\title{
Detecting coronary plaque vulnerability using computed tomography radiomics: the one stop shop for plaque vulnerability?
}

\section{Tim Leiner*}

Utrecht University Medical Center, Department of Radiology, E.01.132, Heidelberglaan 100, 3584CX, Utrecht, The Netherlands

Online publish-ahead-of-print 21 April 2019

\section{This editorial refers to 'Identification of invasive and radio- nuclide imaging markers of coronary plaque vulnerability using radiomic analysis of coronary computed tomography angiography', by M. Kolossváry et al., pp. 1250-1258.}

Advanced atherosclerotic plaques prone to cause acute coronary syndromes (ACS) are characterized by large lipid-rich necrotic cores, increased amounts of inflammatory cells, and thin fibrous caps. These atherosclerotic lesions are designated vulnerable plaques and have a high probability of rupture and atherothrombotic-driven ACS.

An important strategy to lower the incidence of ACS is improved cardiovascular risk assessment. One of the most powerful ways to achieve this goal is by imaging the coronary arteries. Nowadays, there are several intravascular and non-invasive imaging modalities which can be used to detect high-risk coronary plaque characteristics. Serial coronary intravascular ultrasonography (IVUS) is capable of detecting thin-cap fibroatheroma which predispose for myocardial infarction. ${ }^{2}$ Optical coherence tomography (OCT) provides high spatial-resolution images of superficial atherosclerotic plaque microarchitecture and can be used to measure fibrous cap thickness and ulcerations, calcium deposits, fibrous and lipid-rich plaque components, as well as molecular and cellular constituents of plaques. ${ }^{3}$ A non-invasive technique that can identify and localize high-risk coronary plaque is ${ }^{18} \mathrm{~F}$-sodium fluoride PET $\left({ }^{18} \mathrm{~F}-\mathrm{NaF}\right.$ PET $)$. Intense uptake of ${ }^{18} \mathrm{~F}-\mathrm{NaF}$ has been demonstrated in coronary plaques with high-risk features in patients with stable coronary artery disease. ${ }^{4}$ Another non-invasive technique is magnetic resonance imaging. Magnetic resonance imaging can identify endothelial dysfunction, coronary plaque, and coronary intraplaque haemorrhage. ${ }^{5}$

Arguably, the most widely used non-invasive imaging modality to assess coronary plaque in clinical practice today is coronary computed tomography angiography (CCTA). Coronary computed tomography angiography can detect positive remodelling, lowattenuation plaque, and spotty calcification, which are known precursors of ACS. These atherosclerotic plaque characteristics are also associated with myocardial ischaemia and indicative of more malignant, rapidly progressive coronary artery disease. ${ }^{6}$

It is encouraging to see so many diagnostic options to interrogate the different pathophysiological processes underlying plaque formation in the coronary arteries. Nevertheless, no single test is currently capable of capturing all known harbingers of vulnerability in a costeffective and patient friendly manner, leaving physicians managing patients with coronary atherosclerosis with an urgent unmet need.

Kolossváry et al. ${ }^{7}$ present a novel approach to bridge this gap by probing the utility of advanced analysis of the obtained CCTA grey scale values - also known as texture analysis or 'radiomics' - to indicate the presence of different markers of vulnerability as assessed with multiple invasive and non-invasive tests. Radiomics transcends simple visual analysis of computed tomography (CT) images by conversion of digital medical images into high-dimensional data. These data lend themselves to mathematical extraction of advanced quantitative descriptors that are generally not used in clinical practice. This is supplemented by quantification of so-called semantic features, i.e. the terms imagers use to describe lesions. ${ }^{8}$ Radiomics are a powerful set of techniques that have already led to discovery of previously unknown relationships between imaging features, immune response, inflammation, and survival in patients with lung cancer, and Kolossváry et al. are to be commended for extending this methodology to imaging of coronary arteries.

In the present, highly interesting study, 44 coronary plaques from 25 patients with suspected coronary artery disease were analysed. All patients underwent CCTA, ${ }^{18} \mathrm{~F}-\mathrm{NaF}$ PET, IVUS, and OCT within a 90-day period. For all tests, the presence and extent of conventional markers of plaque vulnerability were established and correlated with the presence of at least two conventional features of plaque vulnerability at CCTA. Subsequently, the investigators assessed the correlation between radiomics features and markers of plaque vulnerability. Overall, 935 radiomics features were calculated and the incremental value of these features over conventional features was investigated. The main finding of the study is that radiomics markers significantly improved diagnostic accuracy for detection of vulnerable

The opinions expressed in this article are not necessarily those of the Editors of EHJCl, the European Heart Rhythm Association or the European Society of Cardiology.

* Corresponding author. Tel: +31-88-7556687. E-mail: T.Leiner@umcutrecht.nl

Published on behalf of the European Society of Cardiology. All rights reserved. (c) The Author(s) 2019. For permissions, please email: journals.permissions@oup.com. 
plaque features from CCTA. The highest gain in accuracy was achieved for the radiomics parameter 'surface of high attenuation voxels', which had an area under the receiver operator curve of 0.87 for identification of ${ }^{18} \mathrm{~F}-\mathrm{NaF}$ PET positive lesions vs. 0.65 for conventional high-risk plaque features. Based on these results, the authors correctly conclude that advanced texture analysis of coronary CT images may allow identification of pathophysiological processes from routinely acquired non-invasive coronary CT images that were only thought to be accessible with completely different imaging modalities.

The present study provides us with unique insights. First, it is highly informative to compare different measures of plaque vulnerability in individual patients and to learn to which extent different imaging modalities are complementary. Second, the investigators convincingly show that radiomics analysis greatly increases the amount of information that can be extracted from CCTA, which can provide new insights into its ability to function as a one-stop-shop technique for comprehensive coronary plaque characterization. As such, the investigators have significantly advanced the state-of-the-art. Furthermore, they should be lauded for making available their source code to other interested investigators. ${ }^{10}$

The primary limitations of the study are the small sample size and cross-sectional design. However, it is very difficult to obtain a large cohort of patients willing and able to undergo four imaging tests within a short time. A challenge when dealing with such a small number of patients is to avoid spurious correlations between the large number of radiomics parameters and the small number of conventional vulnerable plaque parameters. In order to deal with this problem, the investigators used stratified cross-validation with a large number of repeats to derive robust estimates of diagnostic performance. This is an accepted way of getting a sense of the generalizability of the diagnostic accuracy in a real-world setting, but-as the investigators note - no substitute for confirmation of the findings in a much larger and independent cohort. In addition, it would be interesting to investigate the evolution of the radiomics parameters over time and in response to initiation of therapy, and to investigate their value for prediction of ACS over established clinical and imaging parameters. Another limitation is the need for manual segmentation of plaques prior to radiomic analysis. This may be overcome by application of deep learning techniques. ${ }^{11,12}$

The results of the present study lay the ground-work for much more detailed analysis of coronary plaque phenotypes. Especially promising in this regard are deep learning techniques that do not rely on hand-crafted features for identification of plaque vulnerability. Preliminary work using such techniques has already demonstrated that deep learning-based analysis can aid in the identification of flowlimiting coronary lesions, ${ }^{13}$ and it is highly likely that identification of vulnerable plaques and patients can be further improved as well.

In conclusion, Kolossváry et al. should be congratulated with this important study that ushers in the era of high dimensional, quantitative coronary plaque analysis in routine clinical practice. The methods described in this study complement clinically used analysis and have the potential to greatly improve individual risk prediction.

Conflict of interest: Grant Support: Philips Healthcare, MR Coils, Medis, Pie Medical. Speakers Bureau: Philips Healthcare, Bayer Healthcare. Patent: T.L. is co-inventor of US patent 10,176,575. The patent is held by Utrecht University Holdings which manages the terms of any licensing agreement.

\section{References}

1. Libby P, Ridker PM, Hansson GK. Progress and challenges in translating the biology of atherosclerosis. Nature 2011;473:317-25.

2. Calvert PA, Obaid DR, O'Sullivan M, Shapiro LM, McNab D, Densem CG et al. Association between IVUS findings and adverse outcomes in patients with coronary artery disease: the VIVA (VH-IVUS in Vulnerable Atherosclerosis) study. JACC Cardiovasc Imaging 2011;4:894-901.

3. Suter MJ, Nadkarni SK, Weisz G, Tanaka A, Jaffer FA, Bouma BE et al. Intravascular optical imaging technology for investigating the coronary artery. JACC Cardiovasc Imaging 2011;4:1022-39.

4. Joshi NV, Vesey AT, Williams MC, Shah AS, Calvert PA, Craighead FH et al. ${ }^{18}$ F-fluoride positron emission tomography for identification of ruptured and high-risk coronary atherosclerotic plaques: a prospective clinical trial. Lancet 2014;383:705-13.

5. Chiribiri A, Botnar RM, Nagel E. Magnetic resonance coronary angiography: where are we today? Curr Cardiol Rep 2013;15:328.

6. Shaw LJ, Blankstein R, Min JK. Outcomes in stable coronary disease: is defining high-risk atherosclerotic plaque important? J Am Coll Cardiol 2019;73:302-4.

7. Kolossváry M, Park J, Bang Jl, Zhang J, Lee JM, Paeng JC et al. Identification of invasive and radionuclide imaging markers of coronary plaque vulnerability using radiomic analysis of coronary computed tomography angiography. Eur Heart J Cardiovasc Imaging 2019;20:1250-58.

8. Gillies RJ, Kinahan PE, Hricak H. Radiomics: images are more than pictures, they are data. Radiology 2016;278:563-77.

9. Grossmann P, Stringfield O, El-Hachem N, Bui MM, Rios Velazquez E, Parmar C et al. Defining the biological basis of radiomic phenotypes in lung cancer. Elife 2017;6:e23421. doi:10.7554/eLife.23421.

10. Kolossváry M. RIA: Radiomics Image Analysis Toolbox for Grayscale Images. 2017. https://CRAN.R-project.org/package=RIA (19 March 2019, date last accessed).

11. Wolterink JM, van Hamersvelt RW, Viergever MA, Leiner T, Išgum I. Coronary artery centerline extraction in cardiac CT angiography using a CNN-based orientation classifier. Med Image Anal 2019;51:46-60.

12. Zreik M, van Hamersvelt RW, Wolterink JM, Leiner T, Viergever MA, Isgum I. A recurrent $\mathrm{CNN}$ for automatic detection and classification of coronary artery plaque and stenosis in coronary CT angiography. IEEE Trans Med Imaging 2018; doi:10.1109/TMI.2018.2883807.

13. Zreik M, Lessmann N, van Hamersvelt RW, Wolterink JM, Voskuil M, Viergever $M A$ et al. Deep learning analysis of the myocardium in coronary CT angiography for identification of patients with functionally significant coronary artery stenosis. Med Image Anal 2018;44:72-85. 